\title{
A Face Recognition System using PCA and Al Technique
}

\author{
Reecha Sharma \\ Department of Electronics and Communication \\ Engineering, Punjabi University, Patiala-147002, \\ Punjab, India.
}

\author{
M.S. Patterh \\ Department of Electronics and Communication \\ Engineering, Punjabi University, Patiala-147002, \\ Punjab, India.
}

\begin{abstract}
In this paper a proficient posture invariant face recognition framework utilizing PCA and AI has been proposed. The peculiarities of an image under test have been extracted utilizing PCA then neuro fuzzy based framework ANFIS is utilized for recognition. The primary reason for this paper is to decrease the computational complexities in the face recognition framework. The proposed framework will perceive the face images under an assortment of stance conditions by utilizing AI based system. The preparation face image dataset will be handled by PCA procedure to register the score esteem, which will be then used in the recognition process. The score values from the distinctive posture images will be given as data to the Neuro-Fuzzy based ANFIS System. The Neuro-Fuzzy based ANFIS System will achieve the recognition transform by taking the info score estimations of the data images and perceive the information face images with the assistance of predefined limit esteem. The proposed face recognition system with Neuro-Fuzzy based ANFIS System will perceive the information face images productively with high recognition proportion. The proposed methodology will be actualized in the MATLAB stage and it will be assessed by utilizing an assortment of database images under different posture invariant conditions. Accordingly, proposed framework will effectively perceive the face images focused around the blend of scores acquired from the posture invariant procedure.
\end{abstract}

\section{Keywords}

Principle Component Analysis (PCA), Face recognition, ANFIS, score value.

\section{INTRODUCTION}

Face recognition is to identify or verify one or more persons in the given still or video images of a scene using a stored database of faces [1]. This is due to its numerous important applications in human-computer interactions, authentication, security, and surveillance [2]. Face recognition can be classified into two categories; they are [4] geometric featurebased and appearance-based. The geometric feature-based methods, such as elastic bunch graph matching [5] and active appearance model [6] make use of the geometrical parameters that measure the facial parts; whereas the appearance-based methods use the intensity or intensity-derived parameters [1]. Face recognition system consists of two stages; they are face detection and the face identification [2]. In the face detection stage, facial images are localized in an input image. In the face identification stage, the localized faces are identified as individuals registered in the system. Therefore, developing both face detection algorithms and face identification algorithms is quite important [11].
The variations involved in face recognition, include illumination, pose, and identity [3], facial expression, hair style, aging, make-up, scale. It is very difficult for even humans to recognize faces correctly when the illumination varies severely, since the same person appears to be very much different [10]. A common solution to handling pose variations in face recognition is the view-based method. In this method, the face images of the individuals to be recognized are acquired from different view angles [13]. The images of the same view are used to construct an Eigen space representation for each view, and the view-specific Eigen space representations are then used for recognizing a person in different poses [12].

However the 2D images patterns of 3D face objects can change dramatically due to lighting and viewing variations [7]. Recently there has been growing interest in face recognition from sets of images. Here, rather than supplying a single query image, the user supplies a set of images of the same unknown individual. In general the gallery also contains a set of images for each known individual, so the system must recover the individual whose gallery set is the best match for the given query set [9]. Recently face recognition using image-set or video sequence has attracted more and more attention within computer vision and pattern recognition community. More importantly, compared with single snapshot, a set or a sequence of images provides much more information about the variation in the appearance of the target subject [8].

The overall structure of the paper is organized as follows: Section 2 reviews the related works with respect to the proposed method. In section 3 motivation of the work is given. In sections 4 , a brief discussion about the proposed methodology is presented, in section 5 analysis the Experimental result and section 6 concludes the paper.

\section{RELATED WORK}

Zhonghua Liu et al. [14] have discussed that the variation of facial expression due to the illumination deteriorates face recognition systems drastically, which is well known as one of the obstacles in face recognition. Yet, the variations of each subject due to the changes of illumination are very analogous to each other. Numerous face classes have been collected, each of which having several images under diverse lighting conditions and obtained a common within-class scatter matrix representing the within-class illumination variations of all the face classes. According to this, an illumination adaptive linear discriminant analysis (IALDA) has been introduced to defeat the illumination variation problems in face recognition when each face class has only one trained sample under the normal lighting conditions. In the IALDA technique, the illumination direction of an input face image has been determined first. 
Subsequently, the respective LDA feature, which is robust to the variations between the images under the estimated lighting conditions and the normal lighting conditions, has been extracted. The potency of the proposed technique has been revealed by conducting experiments on the face databases.

Muhammad Akmal Khan et al. [15] have presented a face recognition approach that improves the face recognition rate than the traditional Principal Component Analysis (PCA). The proposed Sub-Holistic PCA (SH-PCA) technique has been tested using ORL database and outperformed PCA for all test scenarios. Although SH-PCA necessitates more computational power and memory than PCA, it offered an improvement of $6 \%$ accurate recognition on the whole ORL database of 400 images. The SH-PCA technique has achieved $90 \%$ recognition rate for the entire ORL database.

Susheel Kumar et al. [16] have developed an automated system for human face recognition in a real time background world for a giant homemade dataset of persons face. The task was very complicated because the real time background subtraction in an image was still a challenge. Also, there was a huge variation in human face image regarding size, pose, and expression. Thus, AdaBoost with Haar cascade has been employed to identify the real time human face and a simple fast PCA and LDA have been applied to recognize the faces detected. Subsequently, the matched face has been used to mark attendance in the laboratory. This biometric system was a real time attendance system based on the human face recognition with a trouble-free and fast algorithms and yielding a high accuracy rate.

Srinivasan [17] has introduced an adaptive binning and adaboost method based framework for face recognition. Adaptive binning is a robust classifier technique, used to classify the object and the results have been represented in Histogram Gabor Phase Pattern [HGPP]. The resultant HGPP has been again applied with an adaboost classification technique to enhance the competency of the pattern by further diminishing the computational complexity. This framework has been experimentally tested with FERET and found that the recognition rate of the system has been improved substantially. The main feature of this system was a unified model for assessing all the probe sets of the face images and finally, best results have been achieved.

For face recognition, Hafiz Imtiaz et al. [18] have presented a spectral domain feature extraction algorithm that capably uses the local spatial variations in a face image. For feature extraction, here an entropy-based local band selection criterion has been introduced rather than considering the whole face image that selects the high-informative horizontal bands from the face image. Moreover, a feature selection algorithm based on 2D-DFT has been applied to identify the local variations within these high-informative horizontal bands accurately. Magnitudes related to the leading 2D-DFT coefficients have been chosen as features and have shown to offer high within-class compactness and high between-class separability. The dimensionality of the feature space has been diminished by conducting a principal component analysis. Wide experimentations have been performed upon standard face databases and the recognition performance has been compared with some of the prior face recognition approaches. It has been found that the proposed technique can provide computational savings as well as a very high degree of recognition accuracy.

\section{MOTIVATION FOR THE RESEARCH}

The recent research works regarding the face recognition in video or still images are explained briefly in the previous section. From the review, it can be seen that the previous research works have considered various factors like illumination, pose, identity, facial expression, hair style, aging, make-up, scale, etc. But, most of the researches were concentrated on two major factors such as illumination and pose. These existing techniques efficiently perform the face recognition process under these two factors. However, all these works have a drawback in their computational cost, because the variation in illumination results in more computational cost in the face recognition system. Among these two factors, pose is more difficult in face recognition. The requirement to obtain and store a large number of views for each face is a major limitation of post-invariance recognition approach. This technique is inapplicable in circumstances where only a few views of the face to be identified are available. Various algorithms have been developed for face recognition to solve these problems. But, very less works are available regarding the use of pose factor individually. The drawbacks exist in the recognition process are computational complexity, high cost, and huge memory requirements. To overcome these drawbacks, we propose a new face recognition system for images under a variety of pose conditions. The lack of such works in the literature has motivated to do the research in this area.

\section{THE PROPOSED FACE RECOGNITION SYSTEM USING PCA AND ANFIS}

Face recognition is a biological characteristics recognition technology, using the inherent physiological features of humans for ID recognition. In our proposed face recognition technique, the face images gathered from the ORL database. The database face images are denoised using the Adaptive median filter. The denoised image was given to the next process in order to calculate the score value using principle component analysis (PCA) technique. The score value obtained from the PCA technique was given to the ANFIS classifier for accomplishing the training process. Based on the predefined threshold value the image obtained was indicated as recognized or not recognized. For various pose invariant condition the PCA technique and ANFIS classifier perform the face recognition.

The given face database images which are represented as

$$
f_{d}(r, s)=\left\{f_{d^{1}}(r, s), f_{d^{2}}(r, s) \cdots f_{d^{l}}(r, s)\right\} ; i=1,2,3, \cdots N \text {, }
$$

Where $N$ is the total number of images in the database $D$. These numbers of face images from the database $D$ are utilized in the recognition process. The basic architecture of proposed face recognition system is shown in Fig.1. 


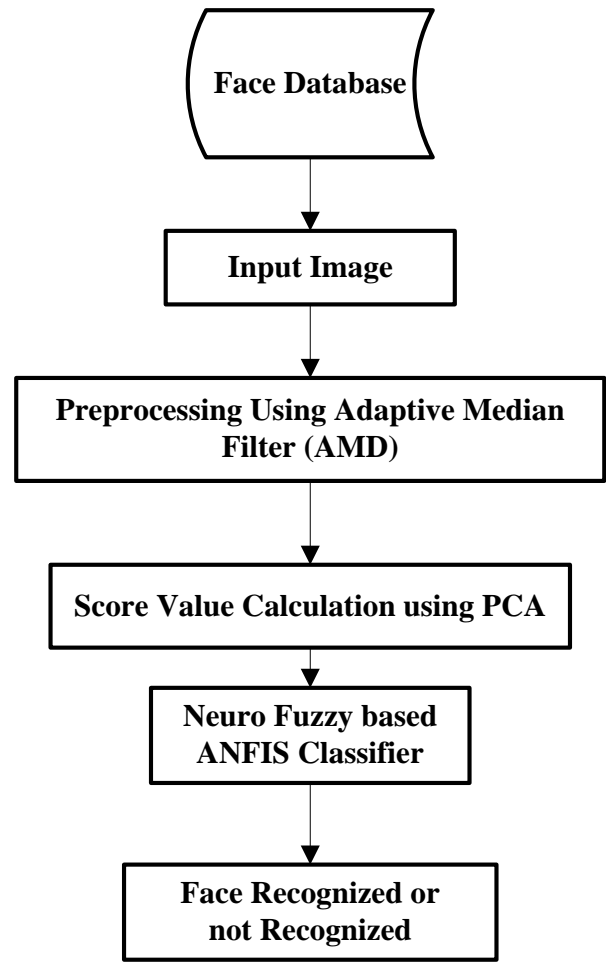

Fig.1. Architecture of the proposed face recognition system

The proposed face recognition technique consists of three stages namely
i) Preprocessing
$>$ Adaptive median filter
ii) Principle component analysis.

Score value calculation

iii) Classification using ANFIS.

\subsection{Adaptive Medan Filter}

The adaptive median filter is applied to the images $f_{d}(r, s)$ which is affected by the (salt and pepper) noise and acquire a noise free image as an output. The process of adaptive median filtering in noise removal is given below,

Step 1: Initialize the window $w$ size $w_{z}$.

Step 2: Check if the center pixel $p_{c e n}(r, s)$ within $w$ is noisy. If the pixel $p_{c e n}(r, s)$ is noisy go to step 3 . Otherwise slide the window to the next pixel and repeat step 1.

Step 3: Sort all pixels within the window $w$ in an ascending order and find the minimum $\left(p_{\min }(r, s)\right)$, median

$\left(p_{\text {med }}(r, s)\right)$, and maximum $\left(p_{\max }(r, s)\right)$ values.

Step 4: Compute if $p_{\text {med }}(r, s)$ is noisy,

$$
\text { (i.e.) }
$$

$p_{\text {min }}(r, s)<p_{\text {med }}(r, s)<p_{\text {max }}(r, s)$

If the median value range is in between the minimum and maximum means the pixel is not a noisy and go to step 5, otherwise $p_{\text {med }}(r, s)$ is a noisy pixel and go to step 6 .
Step 5: Replace the corresponding centre pixel in output image with $p_{\text {med }}(r, s)$ and go to step 8 .

Step 6: Check if all other pixels are noisy. If yes then expend the window size by 2 and go to step 3 . Otherwise, go to step 7.

Step 7: Replace the center pixel of the image with the noise free pixel which is the closest one of the median pixel $p_{\text {med }}(r, s)$.

Step 8: Reset window size $\mathcal{w}_{z}$ and center of window to next pixel.

Step 9: Repeat the steps until all pixels are processed.

Using the above mentioned adaptive median filter algorithm the salt and pepper noise is removed and obtained the noise free image $f_{d}^{\prime}$ thus noise free image is given to the next process to calculate the score value using PCA technique.

\subsection{Dimensionality Reduction using Principle Component Analysis}

The denoised image $f_{d}^{\prime}$ acquired from the Adaptive median filter system is subjected to score esteem estimation utilizing principle component analysis [20].

Steps for calculating the score value of the image:

Step 1: Obtain a set of pixel from an image.

$$
f_{d}^{\prime}=x_{1}, x_{2} \ldots . x_{N}
$$

Where $x_{1}, x_{2} \ldots . . x_{N}$ are the pixel values of image. $\mathrm{N}$ is the total number of pixels in an image.

Step 2: Compute the Mean value.

$$
\mu=(1 / N) \sum_{i=1}^{N} x_{i}
$$

Step 3: Compute the covariance matrix.

$$
K=(1 / N) \sum_{i=1}^{N}\left(x_{i}-\mu\right)\left(x_{i}-\mu\right)^{T}
$$

Step 4: Compute the Eigen vectors $e$ and Eigen values $\lambda$ of the covariance matrix $K$.

If $K$ is a square matrix, a non-zero vector $e$ is an eigenvector of $K$ if there is a scalar $\lambda$ (Eigen value) such that

$$
\text { Solve } K e=\lambda e
$$

Step 5: Arrange eigenvectors in descending order of Eigen values.

$$
\lambda_{1} \geq \lambda_{2} \geq \ldots \ldots \lambda_{n}
$$

The eigenvector with the highest Eigen value is the principle component of the image.

Step 6: PCA reduced the dimension by keeping direction $e$ such that $\lambda<T$

Step 7: Make the reduced set of image features as score values. 
Thus the score value $\boldsymbol{p}\left(\vec{x}_{1}\right), \boldsymbol{p}\left(\vec{x}_{2}\right) \ldots$ $\boldsymbol{P}\left(\overrightarrow{\boldsymbol{x}}_{n}\right)$ thus obtained from the above PCA process for different pose images are then passed into ANFIS classification process.

\subsection{Classification using ANFIS classifier}

The score value $\boldsymbol{p}\left(\overrightarrow{\boldsymbol{x}}_{1}\right), \boldsymbol{p}\left(\overrightarrow{\boldsymbol{x}}_{2}\right)$..

$\boldsymbol{p}\left(\overrightarrow{\boldsymbol{x}}_{n}\right)$ obtained from the PCA are classified using the well known classifier named ANFIS which comprises five layers of nodes. Out of five layers, the first and the fourth layers possess adaptive nodes whereas the second, third and fifth layers possess fixed nodes. The structure of ANFIS having five layered feed-forward neural network is shown in figure 2. In both ANN and FL, the inputs are given to the input layer (as input membership function) and the output is obtained from the output layer (as output membership functions). The architecture of the ANFIS is given in fig.2.

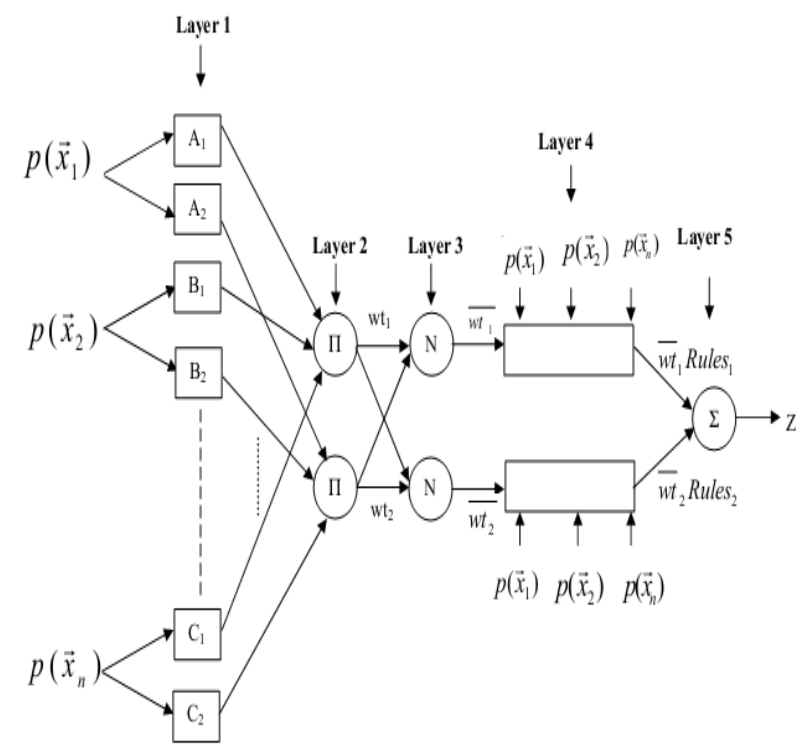

Fig.2. Architecture of ANFIS

The learning process of ANFIS is carried out on the extracted PCA features such as Eigen vectors. The Rule basis of the ANFIS is of the form:

If $\boldsymbol{p}\left(\overrightarrow{\boldsymbol{x}}_{1}\right)$ is $A_{i}, \boldsymbol{p}\left(\overrightarrow{\boldsymbol{x}}_{2}\right)$ is $B_{i}$, is $C_{i}$ then

$$
\text { Rules }_{i}=a_{i} p\left(\vec{x}_{1}\right)+b_{i} p\left(\vec{x}_{2}\right)+c_{i} p\left(\vec{x}_{n}\right)+f_{i}
$$

Where, $\boldsymbol{p}\left(\vec{x}_{1}\right), \boldsymbol{p}\left(\overrightarrow{\boldsymbol{x}}_{2}\right), \boldsymbol{p}\left(\overrightarrow{\boldsymbol{x}}_{n}\right)$ are the inputs, $A_{i} B_{i}$ and $C_{i}$ are the fuzzy sets, Rules ${ }_{i}$ is the output within the fuzzy region specified by the fuzzy rule, $a_{i}, b_{i}, c_{i}$ and $f_{i}$ are the design parameters that are determined by the training process.

Layer 1: Every node $i$ in this layer is a square node with a node function.
$O_{1, i}=\mu_{A_{i}}\left(p\left(\vec{x}_{1}\right)\right), O_{1, i}=\mu_{B_{i}}\left(p\left(\vec{x}_{2}\right)\right), O_{1, i}=\mu_{C}\left(p\left(\vec{x}_{n}\right)\right)$

Usually $\mu_{A_{i}}\left(p\left(\vec{x}_{1}\right)\right), \mu_{B_{i}}\left(p\left(\vec{x}_{2}\right), \mu_{C_{i}}\left(p\left(\vec{x}_{n}\right)\right)\right.$ are chosen to be bell-shaped with maximum equal to 1 and minimum equal to 0 and are defined as

$$
\mu_{A_{i}}\left(p\left(\vec{x}_{1}\right)\right)=\mu_{B_{i}}\left(p\left(\vec{x}_{2}\right)\right)=\mu_{C}\left(p\left(\vec{x}_{n}\right)\right)=\frac{1}{1+\left[\left(\frac{x-o_{i}}{p_{i}}\right)^{2}\right]^{q_{i}}}
$$

Where $o_{i}, p_{i}, q_{i}$ is the parameter set. These parameters in this layer are referred to as premise parameters.

Layer-2: Every node in this layer is a circle node labeled $\Pi$ which multiplies the incoming signals and sends the product out. For instance,

$O_{2, i}=w t_{i}=\mu_{A_{i}}\left(p\left(\vec{x}_{1}\right)\right) \times \mu_{B_{i}}\left(p\left(\vec{x}_{2}\right)\right) \times \mu_{C_{i}}\left(p\left(\vec{x}_{n}\right)\right), \quad i=1,2$

Each node output represents the firing strength of a rule.

Layer-3: Every node in this layer is a circle node labeled $N$. The $i^{t h}$ node calculates the ratio of the $i^{\text {th }}$ rules firing strength to the sum of all rule's firing strengths:

$O_{3, i}=\overline{w t}_{i}=w t_{i} /\left(w t_{1}+w t_{2}\right), \quad i=1,2$

Layer-4: Every node $i$ in this layer is a square node with a node function

$$
O_{4, i}=\overline{w t}_{i} \cdot \text { Rules }_{i} \quad i=1,2
$$

Where $w t_{i}$ is the output of layer 3 and $a_{i}, b_{i}, c_{i}, f_{i}$ are the parameter set. Parameters in this layer will be referred to as consequent parameters.

Layer-5: The single node in this layer is a circle node labeled $\sum$ that computes the overall output as the summation of all incoming signals:

$$
\begin{aligned}
& O_{5, i}=\sum_{i} \overline{w t}_{i} \text { Rules }_{i}=\frac{\sum_{i} w t_{i} \text { Rules }_{i}}{\sum_{i} w t_{i}} \\
& Z=\frac{w t_{1} \text { Rules }_{1}+w t_{2} \text { Rules }_{2}}{w t_{1}+w t_{2}}
\end{aligned}
$$

$$
Z=\overline{w t} \text { Rules }_{1}+\overline{w t} \text { Rules }_{2}
$$

Then the predefined threshold value $\omega$ and the result of the neural network $(Z)$ is compared which is given in Eq. (17). result $=\left\{\begin{array}{l}\text { recognized }, Z \geq \omega, \\ \text { not recognized }, Z<\omega\end{array}\right.$ 
The neural network output is greater than the threshold value $\omega$

means, the given input image is recognized or the image is unrecognized. Thus the ANFIS is well trained using the score value obtained from PCA. The ANFIS classifier indicates whether the image is recognized or not recognized.

The performance of the well trained ANFIS is tested by giving more number of different pose images.

\section{EXPERIMENTAL RESULTS}

The proposed PCA based face detection technique with the aid of ANFIS for different pose images was implemented in the working platform of MATLAB (version 7.12) with machine configuration as follows.

Processor: Intel core i7

OS: Windows 7

CPU speed: $3.20 \mathrm{GHz}$

\section{RAM: 4GB}

The performance of the proposed PCA based face detection technique for different pose images are evaluated by giving more number of images taken from the ORL database. Fig.3 shows some sample images fetched from the database.
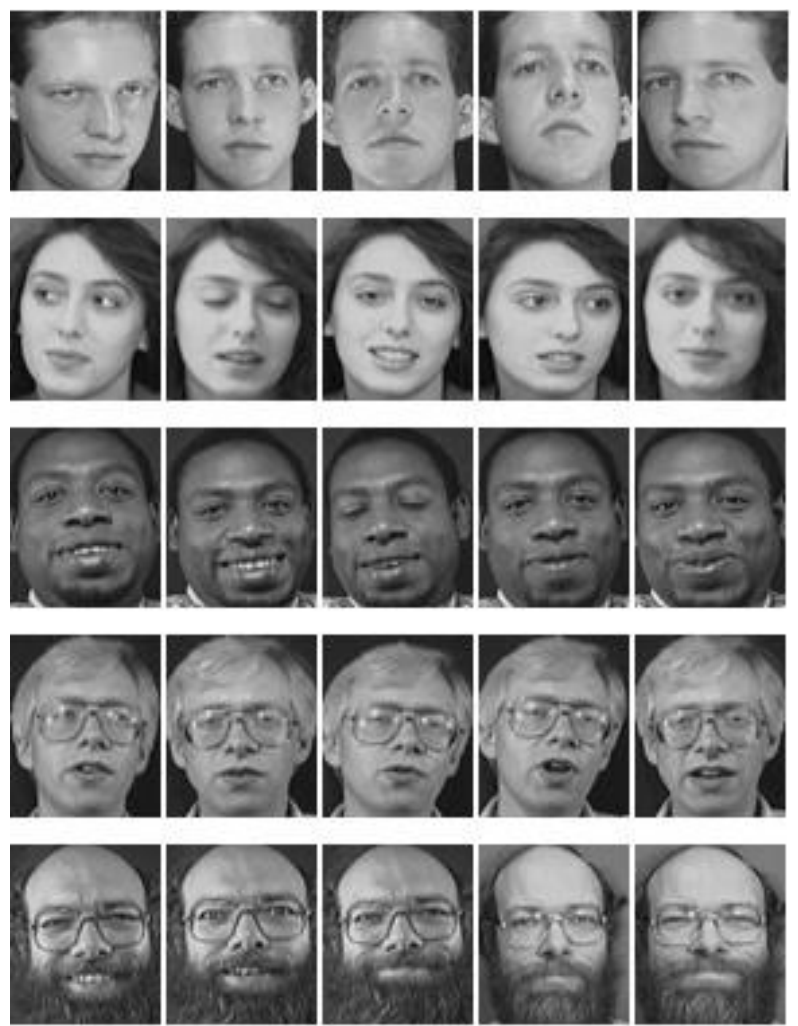

Fig.3. Sample 5 dataset from the ORL database

To remove the noise from the given input face images, the images are given to the preprocessing process by using the Adaptive median filtering and that noise removed face images are shown in fig.4.
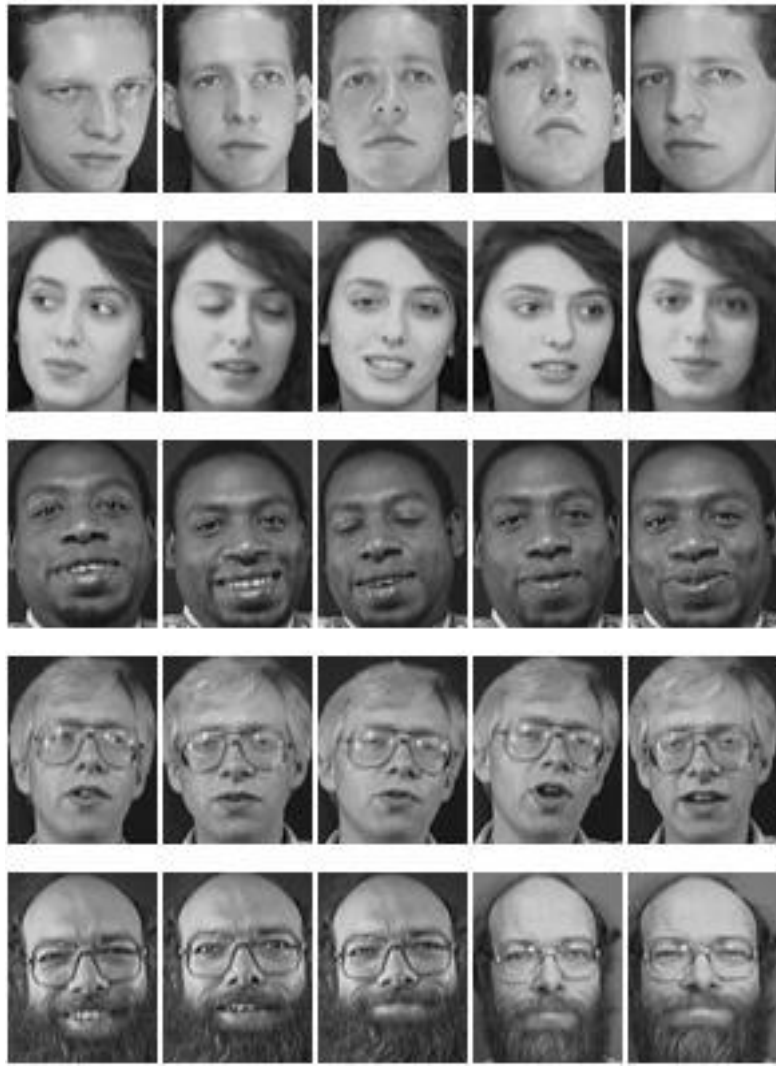

Fig.4. Preprocessed images using Adaptive median filtering

Accordingly the denoised image acquired from the Adaptive median filter is given to the following methodology to compute the score value utilizing the PCA based calculation. The score esteem in this way acquired from the principle component analysis was given as the information to the ANFIS classifier. The ANFIS classifiers perceive the face image proficiently. In testing more number of face images are used to examine the execution of our proposed face recognition system.

\section{PERFORMANCE ANALYSIS}

By applying the statistical measures the performance of proposed face recognition system is examined. The face image from ORL database are utilized to analyze the performance of proposed PCA technique with the existing ICA, LDA and Existing [19] techniques to detects the faces rapidly. The comparison result of the proposed technique with the existing ICA, LDA and Existing [19] techniques has shown in the table 1.Similarly the proposed Adaptive median filter and it is shown in table 2. filter is compared with the Existing Average and Gaussian.

Table 1: Demonstrate the Performance measure of the proposed PCA technique with the ICA, LDA and Existing technique

\begin{tabular}{|c|c|c|c|c|}
\hline Measures & proposed & ICA & LDA & $\begin{array}{c}\text { Existing } \\
\text { technique[19] }\end{array}$ \\
\hline Accuracy & 0.9666 & 0.713 & 0.68 & 0.96555 \\
\hline Sensitivity & 0.9729 & 0.728 & 0.6483 & 0.5768 \\
\hline Specificity & 0.9605 & 0.712 & 0.7288 & 0.80657 \\
\hline
\end{tabular}


Discussion: In table 1 the proposed PCA technique is compared with the ICA, LDA and Existing technique [19] techniques in terms of accuracy sensitivity and specificity measures. From the above table the accuracy of the proposed method is (0.9666) but the ICA, LDA and Existing technique [19] has offer only (0.713), (0.68), (0.96555) of accuracy. Similarly the sensitivity and specificity of the proposed method is (0.9729) and (0.9605) but the ICA, LDA and Existing technique [19] gives (0.728), (0.6483), (0.5768) of sensitivity and (0.712), $(0.728(0.80657)$ of specificity respectively. Even though the accuracy of the proposed PCA method and the existing technique [19] is probably equal, the sensitivity and the specificity of the existing [19] is very low when compared to the proposed technique Hence from the table it has been prove that our proposed method has recognized the image more accurately.

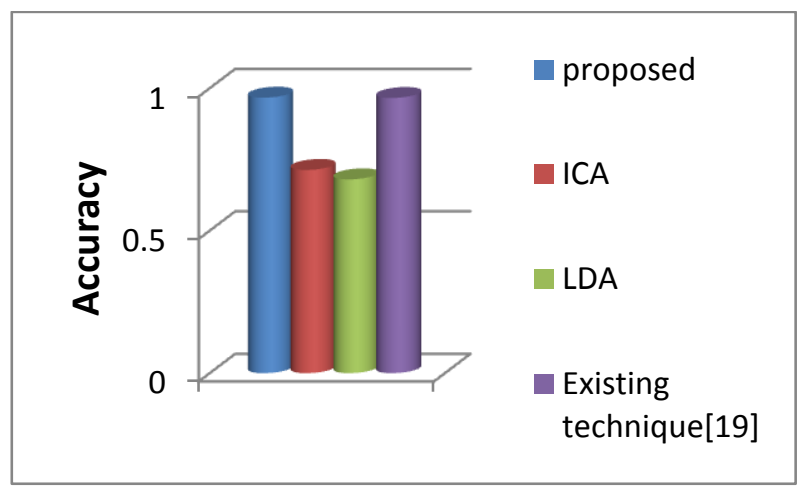

(I)

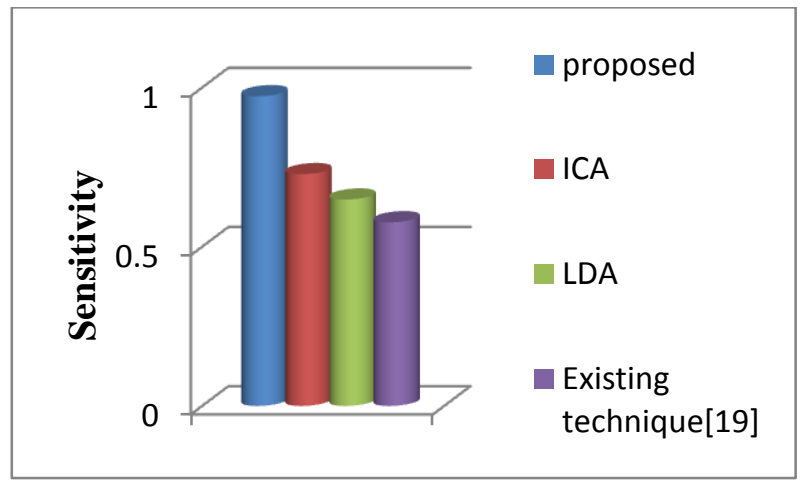

(II)

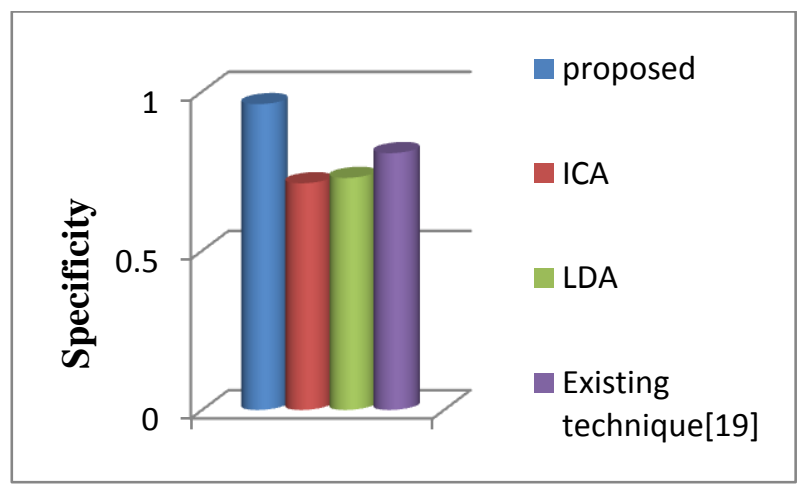

(III)

Fig.5. Illustrate the performance of the proposed PCA technique with the ICA LDA and the Existing method
Fig.5 demonstrates the performance of the proposed PCA technique with the ICA, LDA and the Existing method [19] in terms of accuracy sensitivity and specificity. From the fig.5. (i) the accuracy of the proposed method high when compared to ICA, LDA and probably equal Existing method [19].Also the sensitivity and the specificity of the existing [19] is low when compared to the proposed technique. Hence from the performance graph it has been prove that proposed method has recognized the image more precisely and effectively.

Table 2: Image denoising performance of our proposed adaptive median filter and existing Average and Gaussian filtering methods

\begin{tabular}{|c|c|c|c|}
\hline \multirow{2}{*}{ Images } & \multicolumn{3}{|c|}{ PSNR } \\
\cline { 2 - 4 } & $\begin{array}{c}\text { Proposed } \\
\text { adaptive } \\
\text { median } \\
\text { filter }\end{array}$ & $\begin{array}{c}\text { Existing } \\
\text { Average } \\
\text { Filter } \\
\text { (in dB) }\end{array}$ & $\begin{array}{c}\text { Existing } \\
\text { Gaussian Filter } \\
\text { (in dB) }\end{array}$ \\
\hline $\mathbf{1}$ & 38.64005 & 28.37 & 26.35 \\
\hline $\mathbf{2}$ & 33.977 & 26.28 & 24.41 \\
\hline $\mathbf{3}$ & 35.1861 & 26.81 & 26.02 \\
\hline $\mathbf{4}$ & 34.54 & 26.19 & 25.52 \\
\hline $\mathbf{5}$ & 33.96 & 26.68 & 25.08 \\
\hline
\end{tabular}

Discussion: As can be seen from the table 2, proposed Adaptive median filter with PCA has attained high denoising ratio than the other filtering methods. Proposed Adaptive median filter has given high PSNR value for different dataset images. When compared to the existing Average and Gaussian filtering methods. The comparison graph is shown in fig.6. for the proposed Adaptive median filtering method and the existing average and Gaussian filtering method in terms of the PSNR values.

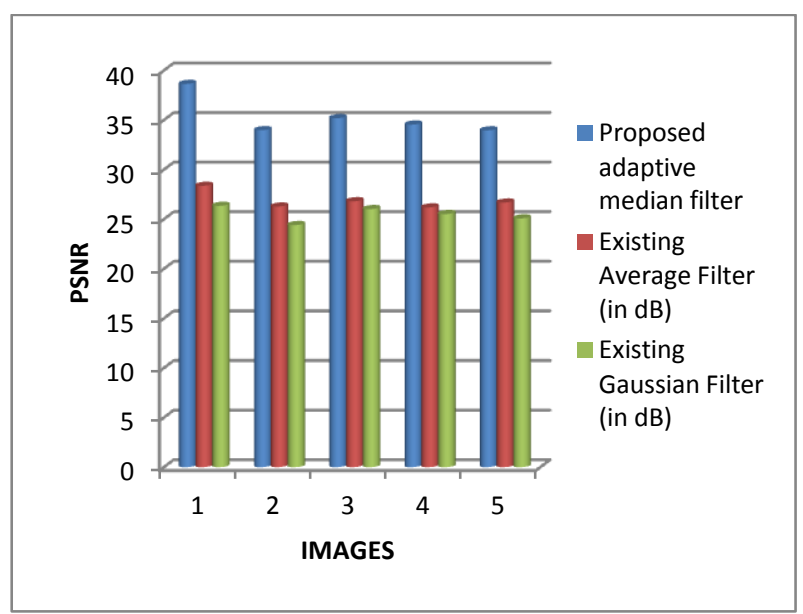

Fig.6. Illustrate the proposed Adaptive median filtering technique with the existing average and Gaussian filtering methods. 
Discussion: From the fig.6 it has been shown that the PSNR value of the proposed Adaptive median filtering method is higher than the existing average and Gaussian filtering methods hence from the performance analysis graph it has been clear that our proposed technique remove the noise efficiently than any other existing techniques.

Moreover proposed classifier ANFIS is compared with the existing technique such as FFBNN in terms of sensitivity, specificity and accuracy measures. The performance result of comparison techniques has been shown below in table 3 .

Table 3: Performance measures of the proposed ANFIS technique and the existing FFBNN techniques in terms of accuracy, sensitivity, specificity.

\begin{tabular}{|c|c|c|}
\hline Measures & Proposed ANFIS & Existing FFBNN \\
\hline Accuracy & 0.9666 & 0.8666 \\
\hline Sensitivity & 0.9729 & 0.8481 \\
\hline Specificity & 0.9605 & 0.8873 \\
\hline FPR & 0.0394 & 0.1126 \\
\hline PPV & 0.96 & 0.8933 \\
\hline NPV & 0.9733 & 0.84 \\
\hline FDR & 0.04 & 0.106 \\
\hline MCC & 0.9334 & 0.7343 \\
\hline
\end{tabular}

Discussion: In table 3 the proposed ANFIS classifier is compared with the existing FFBNN classifier in terms of accuracy, sensitivity and specificity measures. From the table 3 it has been shown that the proposed ANFIS has given of (0.9666) accuracy but the existing FFBNN has given only (0.8666) of accuracy respectively. Similarly the sensitivity and the specificity of proposed method are higher than the existing FFBNN. The comparison graph has been shown in fig.7.

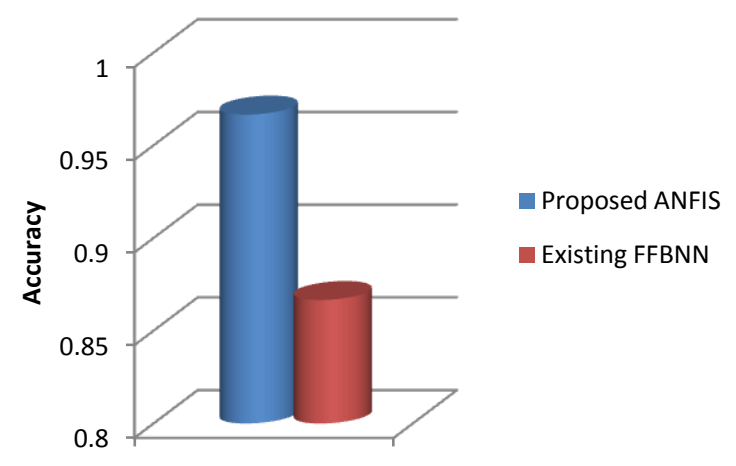

(I)

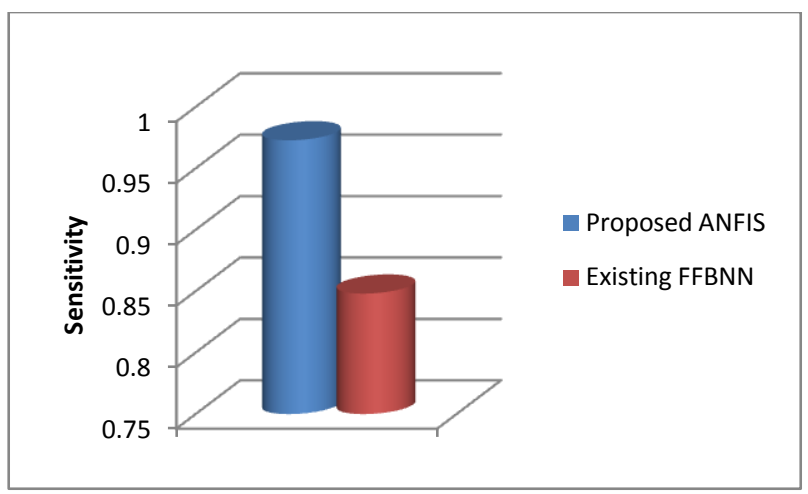

(II)

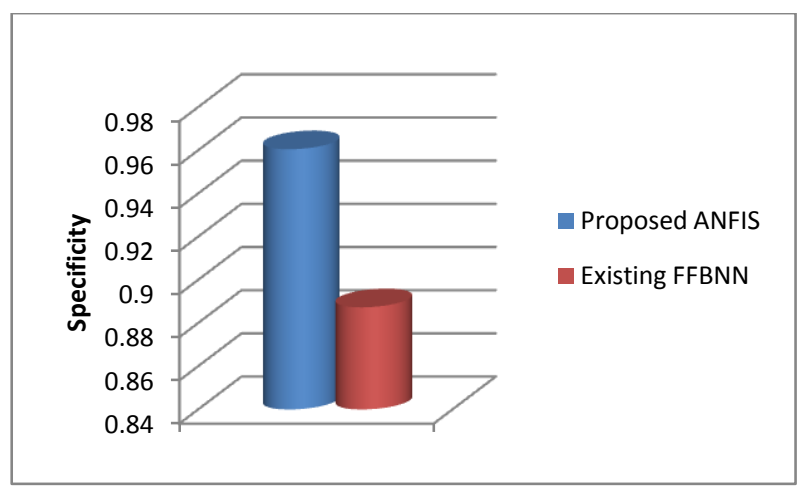

(III)

Fig.7. Comparison of proposed ANFIS technique with the existing FFBNN in terms of accuracy, sensitivity and specificity measures

Fig.7 has shown the comparison graph of accuracy, sensitivity and specificity of the proposed PCA based technique with the existing FFBNN technique. From the Fig. 7 it has been prove that the performance measure of the proposed classifier is high when compared to the existing FFBNN classifier. Thus from the performance metrics it has been shown that the proposed classifier efficiently recognize the images.

\section{CONCLUSION AND FUTURE SCOPE}

In this paper, a face recognition technique with the aid of ANFIS has been proposed. The proposed technique was implemented on ORL database and a huge set of test analysis were utilized to analyze the outcomes of the proposed face recognition technique. It offers a significant tempo of recognition rate with less false positive rate. The comparison result shows that proposed face recognition technique based on ANFIS has given high recognition rate than the ICA, LDA and FFBNN existing technique. The sensitivity and specificity rate of proposed technique is also high as compare to ICA, LDA. In future this technique can be enhanced for illumination invariant face recognition.

\section{ACKNOWLEDGMENTS}

I would like to express my greatest gratitude to my guide Dr. M.S Patterh Department of ECE, Punjabi University for his continuous support for the paper from initial advice \& contacts in the early stages of conceptual inception \& through ongoing advice \& encouragement to this day. I would like to thank the anonymous reviewers for their constructive comments 


\section{REFERENCES}

[1] Wu-Jun Li, Chong-Jun Wang, Dian-Xiang Xu and Shi-Fu Chen, "Illumination Invariant Face Recognition Based on Neural Network Ensemble", In Proceedings of 16th IEEE International Conference on Tools with Artificial Intelligence, pp. 486-490, November 2004

[2] Ehab F. Abdel-Kader, Rabab M. Ramadan and Rawya Y. Rizk, "Rotation Invariant Face Recognition Based on Hybrid LPT/DCT Features", International Journal of Electrical and Computer Engineering, Vol. 3, No. 7, pp. 488-493, 2008

[3] Shaohua Kevin Zhou and Rama Chellappa, "Image-based face recognition under illumination and pose variations", Journal of the Optical Society of America A, Vol. 22, No. 2, pp. 217-229, 2005

[4] Brunelli and Poggio, "Face recognition: Features versus templates", IEEE Transactions on Pattern Analysis and Machine Intelligence, Vol. 15, No. 10, pp.1042-1052, 1993

[5] Laurenz Wiskott, Jean-Marc Fellous, Norbert Kruger, and Christoph von der Malsburg, "Face Recognition by Elastic Bunch Graph Matching", IEEE Transactions on Pattern Analysis and Machine Intelligence, Vol.19, pp. 775-779, 1997

[6] Edwards, Cootes and Taylor, "Face recognition using active appearance models", In Proceedings of the 5th European Conference on Computer Vision, Vol. 2, Freeburg, Germany, pp. 581-595, 1998

[7] WenYi Zhao and Rama Chellappa, "Image based Face Recognition Issues and Methods", Image Recognition and Classification, pp. 375-402, 2002

[8] Xi Li, Kazuhiro Fukui and Nanning Zheng, "Image-set based Face Recognition Using Boosted Global and Local Principal Angles", Springer Lecture Notes in Computer Science (LNCS), 2009

[9] Hakan Cevikalp and Bill Triggs, "Face Recognition Based on Image Sets", In Proceedings of the IEEE Conference on Computer Vision and Pattern Recognition, San Francisco : United States, 2010

[10] Seok Cheol Kee, Kyoung Mu Lee And Sang Uk Lee, " Illumination Invariant Face Recognition Using Photometric Stereo", IEICE Transactions on Information and Systems, Vol. E83-D, No. 7, pp. 1466-1474, July 2000

[11] Yasufumi Suzuki and Tadashi Shibata, "IlluminationInvariant Face Identification Using Edge-Based Feature Vectors In Pseudo-2d Hidden Markov Models", In Proceedings of the 14th European Signal Processing Conference, Florence, Italy, 2006

[12] Hui-Fuang Ng, "Pose-Invariant Face Recognition Security System", Asian Journal of Health and Information Sciences, Vol. 1, No. 1, pp. 101-111, 2006

[13] Shermina, "Impact of Locally Linear Regression and Fisher Linear Discriminant Analysis in Pose Invariant Face Recognition", International Journal of Computer
Science and Network Security, VOL.10 No.10, pp. 106110 , October 2010

[14] Zhonghua Liu, Jingbo Zhou and Zhong Jin, "Face recognition based on illumination adaptive LDA", In Proceedings of International Conference on Pattern Recognition, pp. 894-897, Istanbul, Turkey, August 2010

[15] Muhammad Akmal Khan, Javed and Muhammad Anjum Akbar, "Face Recognition using Sub-Holistic PCA", British Journal of Science, Vol. 1, No. 1, pp. 111-120, 2011

[16] Susheel Kumar, Shitala Prasad, Vijay Bhaskar Semwal and Tripathi, "Real Time Face Recognition using Adaboost Improved Fast PCA Algorithm", International Journal of Artificial Intelligence \& Applications (IJAIA), Vol.2, No.3, July 2011

[17] Srinivasan, "A Framework for Face Recognition Using Adaptive Binning and Adaboost Techniques", The International Journal of Multimedia \& Its Applications, Vol.3, No.1, pp. 76-88, 2011.

[18] Hafiz Imtiaz and Shaikh Anowarul Fattah, "A Spectral Domain Local Feature Extraction Algorithm for Face Recognition", International Journal of Security (IJS), Vol. 5, No. 2, pp. 62-73, 2011.

[19] Shermina and Vasudevan "An Efficient Face Recognition System Based On the Fusion of MPCA and LPP", American Journal of Scientific Research, No. 11, pp. 619,2010

[20] Syed Navaz, Dhevi Sri and Pratap Mazumder, "Face Recognition Using Principal Component Analysis And Neural Networks", International Journal of Computer Networking, Vol. 3, No. 1, pp. 245-256, 2013.

\section{AUTHOR PROFILE}

Reecha Sharma is doing Ph.d in the field of Image Processing. She did her B.Tech. in Electronics and Instrumentation Engineering from MMEC Mullana, Ambala, India in 2003. She did M.E in Electronics, Instrumentation and Control Engineering from Thapar University Patiala, India in 2005.She has nine years of teaching experience. She has guided fifteen M.Tech. students. She has published over 30 research papers in International/National Journals and Conferences. Her area of research is Image Processing. At present she is working as Assistant Professor in Department of ECE, Punjabi University Patiala, Punjab, India.

M. S. Patterh, did his graduation in Electronics Engineering in 1988 from MITS Gwalior India, Masters from BITS Pilani India in 1993 and $\mathrm{Ph} \mathrm{D}$ in Electronics and Communication from PTU India. He is having over 23 years of teaching and over 13 years of research experience. At present he is working as Professor in Department of ECE, Punjabi University Patiala, India. His areas of interest include Signal Processing and Communication Engineering. He has published over 100 research papers in International/National Journals and Conferences. He is awarded twice Sir Thomas Memorial Ward prize by Institution of Engineers (India) for the research contributions in the area of Digital Communications. He has supervised six $\mathrm{PhDs}$ to date. He is member of IEEE, IETE, IE(I) and LSTE. He has completed projects worth $\$ 100,000$. 